\title{
Time course of improvement with antipsychotic medication in treatment-resistant schizophrenia
}

Takefumi Suzuki, Gary Remington, Tamara Arenovich, Hiroyuki Uchida, Ofer Agid, Ariel Graff-Guerrero and David C. Mamo

\section{Background}

Improvements are greatest in the earlier weeks of antipsychotic treatment of patients with non-resistant schizophrenia.

\section{Aims \\ To address the early time-line for improvement with antipsychotics in treatment-resistant schizophrenia.}

\section{Method}

Randomised double-blind trials of antipsychotic medication in adult patients with treatment-resistant schizophrenia were investigated (last search June 2010). A series of metaregression analyses were carried out to examine the effect of time on the average item scores in the Positive and Negative Syndrome Scale (PANSS) or Brief Psychiatric Rating Scale (BPRS) at three or more distinct time points within the first 6 weeks of treatment.

\section{Results}

Study duration varied from 4 weeks to 1 year and the definitions of treatment resistance as well as of treatment response were not necessarily consistent across 19 identified studies, resulting in highly variable rates of response (0-76\%) The mean standardised baseline item score in the PANSS or BPRS was 3.4 (s.e. $=0.06$ ) in the five studies included in the meta-regression analysis, with the average baseline Clinical Global Impression - Severity score being 5.2 (marked illness). For the pooled population treated with a range of antipsychotics $(n=1019)$, significant reductions in the mean item scores occurred during the first 4 weeks; improvements observed in later weeks were smaller and non-significant. In contrast, weekly improvement with clozapine was significant throughout $(n=356)$.

\section{Conclusions}

Our findings provide preliminary evidence that the majority of improvement with antipsychotics may occur relatively early. More consistent improvements with clozapine may be associated with a gradual titration. To further elucidate response patterns, future studies are needed to provide data over regular intervals during earlier stages of treatment.

\section{Declaration of interest}

T.S. has received fellowship grants from the Government of Canada Post-Doctoral Research Fellowships, Japanese Society of Clinical Neuropsychopharmacology, Kanae Foundation and Mochida Memorial Foundation, and manuscript fees from Dainippon Sumitomo Pharma and Kyowa Hakko Kirin. G.R. has received research support from the Schizophrenia Society of Ontario and the Canadian Diabetes Association. As a principal investigator he has also received support from Novartis Canada and Medicure. As a co-investigator he has received research support from the Canadian Institutes of Health Research and the Canadian Psychiatric Research Foundation. He is a co-investigator in research sponsored by Pfizer. He has received consultant fees from CanAm Bioresearch as well as speaker's fees from Novartis. H.U. has received grants, speaker's honoraria or manuscript fees from Pfizer Health Research Foundation, GlaxoSmithkline, Otsuka Pharmaceutical, Dainippon Sumitomo Pharma, Janssen Pharmaceutical and Pfizer. O.A. has served as or received advisory board/speaker honoraria from Eli Lilly and Janssen-Ortho and has a research contract with Pfizer. A.G.-G. has received grants or consultation fees from Abbott Laboratories, Janssen-Cilag and Lilly. D.C.M. has received grants or consultant fees from Bristol-Myers Squibb and Pfizer and has received speaker's honoraria from AstraZeneca.
Recent reports have indicated that antipsychotic drugs exert their clinical effects earlier than previously thought, with the largest treatment effect occurring within 2-4 weeks, which has challenged the concept of delayed onset of action of antipsychotics. ${ }^{1,2}$ However, none of these studies investigated time to response in treatment-resistant schizophrenia. This is clinically important, since it is known that such patients constitute about a third of treated cases. ${ }^{3}$ Moreover, these individuals are often subjected to multiple trials of antipsychotic drugs, including clozapine, without clear guidance as to the trial duration that could be considered adequate prior to moving to the subsequent strategies. For instance, time to response remains a controversial issue even with clozapine, a gold standard antipsychotic medication in treatment-resistant schizophrenia. ${ }^{4,5}$ At least one shorter-term investigation has demonstrated that a degree of improvement in Brief Psychiatric Rating Scale (BPRS) scores at 1 week can predict favourable outcome at 5 weeks. ${ }^{6}$ Further, some longer-term studies are not supportive of treatment response beyond 6-8 weeks. $^{7,8}$ Nevertheless, another report showed that $55 \%$ of treatment response occurred after 6 weeks in individuals treated for a mean of 10 months (s.d.=8). ${ }^{9}$ In addition, Meltzer et al reported that 20 of 36 participants classified as responders (response defined as more than 20\% decrease in BPRS score) were identified after 6 weeks of treatment with clozapine. ${ }^{10}$ A majority of treatment response $(70 \% ; n=14)$ was also attained later than 6 weeks if a threshold of response was set more stringently (i.e. a $50 \%$ or more decrease in BPRS score). Moreover, Zito et al reported that although $19 \%$ of patients exhibited improvement at 6 weeks, the response rate increased to $29 \%$ at 12 weeks. ${ }^{11}$ Indeed, one guideline recommends treatment to be continued for at least 8 weeks. ${ }^{12}$ However, clinicians have no guidance on when to consider a clozapine trial as failed, a question of major clinical relevance considering the adverse effects profile of this medication. We therefore conducted a systematic review of the literature in treatment-resistant schizophrenia to address response rates and time-line to response, focusing specifically on early improvement in this population.

\section{Method}

A literature search was conducted systematically to identify randomised, double-blind clinical trials of antipsychotics in adult 
patients with treatment-resistant schizophrenia. PubMed, Ovid Medline (since 1950), EMBASE (since 1980) and PsycINFO (since 1967) through June 2010 were searched with key words 'treatment resistant schizophrenia' AND 'double blind'. A search was also conducted by replacing 'treatment resistant schizophrenia' with 'refractory schizophrenia'. Studies with fewer than ten adult patients in the treatment arms, studies for which response rate was not reported and dose-finding studies were excluded. Likewise, augmentation studies after run-in antipsychotic treatment were excluded.

\section{Qualitative analysis}

Each study was examined in terms of definition of treatment resistance. Definition of treatment response, rate of response, premature attrition rate and data reporting points were also investigated, to give an indication of time to antipsychotic response in treatment-resistant schizophrenia.

\section{Statistical analysis}

To investigate potentially early treatment improvement or onset of action, publications that reported at least three last observation carried forward (LOCF) data points in the Positive and Negative Syndrome Scale (PANSS; each item scored 1-7) or BPRS (each item scored 1-7) up to the first 6 weeks of treatment were extracted and mean item scores calculated by dividing the total score by 30 or 18 respectively. This method is in accordance with a previous investigation of early response to antipsychotics in non-resistant schizophrenia, ${ }^{2}$ and allows for the relative scarcity of available data points (see Results). The LOCF scores during the first 6 weeks of treatment were either reported as absolute scores in the original article (1 study), ${ }^{13}$ obtained by the authors on request ( 1 study), ${ }^{14}$ or determined from the accompanying figures detailing change over this period ( 3 studies $)^{5,15,16}$ in the studies included in our meta-regression analyses. Data solely concerning clozapine were considered separately. Also, data on second-generation antipsychotics (other than clozapine) and study completer groups were investigated for descriptive purposes only.

A series of meta-regressions were carried out (via weighted mixed models analyses) using SAS version 9.1.3. for Windows to establish the time course of treatment response among participants with treatment-resistant schizophrenia. These models make less restrictive assumptions than many standard statistical tests (i.e. we need not to assume that our residuals are independent when using mixed models) and are appropriate for use when studying longitudinal data. Full maximum likelihood estimation was selected as the method of estimation for each of these analyses, and a variety of potential residual covariance structures (variance components, first-order autoregressive, compound symmetric, heterogeneous first-order autoregressive, heterogeneous compound symmetric, and unstructured) were investigated in each model. Although the structure and interpretation of the residual covariance matrix are of little clinical interest and do not relate directly to our research questions, we chose to investigate this series of modelling assumptions in order to identify the models and series of assumptions that best fit our data. This in turn improved the precision of our estimates (i.e. reduced the magnitude of standard errors in the estimates), which affects hypothesis testing related to our research questions.

To determine objectively which of these structures most accurately represents the true residual covariance structure within each of our investigations, we chose to examine the Bayesian information criteria (BIC) associated with each model - a goodness-of-fit statistic that penalises the model's likelihood function based on both model complexity (the number of parameters requiring estimation) and sample size. Use of this statistic allows us to identify the most parsimonious model (the model requiring estimation of the fewest parameters) which best fits our sample data. A smaller BIC indicates a better fit when comparing models, and the covariance structure ultimately adopted was the one that yielded the smallest BIC value for each of our analyses.

As one cannot reasonably assume independence of results across study arms within the same study, the findings for each of the two drug treatment groups within a given study were pooled to reflect a single set of results for each study, using a weighted average of the standardised scores across the two drug groups at each time point. Baseline sample sizes (pooled across treatment arms) for each study were used as sample weights in all analyses.

Percentage reduction in PANSS scores was obtained by taking into account the non-deductible value of the scale (i.e. 30$) ;{ }^{17}$ for instance, if the baseline mean item score was 4.0 (120 in total), a $20 \%$ reduction was defined as a post-treatment mean item score of 3.4, i.e. a total score of 102 ; note that $(102-120) /(120-30)$ equals -0.2 .

\section{Results}

The literature search using the key words 'treatment resistant schizophrenia' and 'double blind' located 14 studies and a replacement of the former search term with 'refractory schizophrenia' found five studies (Fig. 1). In total, 19 manuscripts reporting response rates following antipsychotic treatment of treatment-resistant/refractory schizophrenia in adult patients were identified (online Tables DS1 and DS2). ${ }^{5,13,14-16,18-31}$ The definition of treatment resistance varied among studies, although it invariably included past failure to respond to one or more antipsychotics at chlorpromazine equivalent doses of $400-1000 \mathrm{mg}$. Similarly, the definitions of treatment response were not necessarily consistent, although they universally adopted a specified degree of improvement in the representative rating scales (typically a $20-30 \%$ decrease in PANSS or BPRS score).

Study duration varied from 4 weeks to 1 year, with the majority (63\%) lasting for 12 weeks or less (12 of 19 studies). In the light of these differences it is not surprising to note that both response rates and premature attrition rates were variable (0-76\% and $2-72 \%$ respectively). A total of 11 antipsychotics were included in these studies, with the most frequently studied being clozapine (ten studies), haloperidol (seven), chlorpromazine (five), olanzapine (five) and risperidone (four). Only nine reports $(47 \%)$ described data on key outcomes throughout available points; for the remainder, data were either unclear regarding assessment points, were selectively reported or were not fully provided throughout the treatment period (e.g. pre- and posttreatment presentation only). Only one study explicitly reported the rate of response through week 6 (excepting week 5) ${ }^{15}$ Results of this study showed a continuous increase in the percentage of responders (observed cases) for aripiprazole, whereas the slope of increase was more gradual for perphenazine after 3 weeks. In contrast, LOCF data from an intent-to-treat (ITT) sample implied the largest decrease in average PANSS scores in the first 3-4 weeks.

\section{Meta-regression analysis}

\section{Studies included}

One complex study that adopted a multiple transition among antipsychotics was excluded from the meta-regression analysis. ${ }^{20}$ 


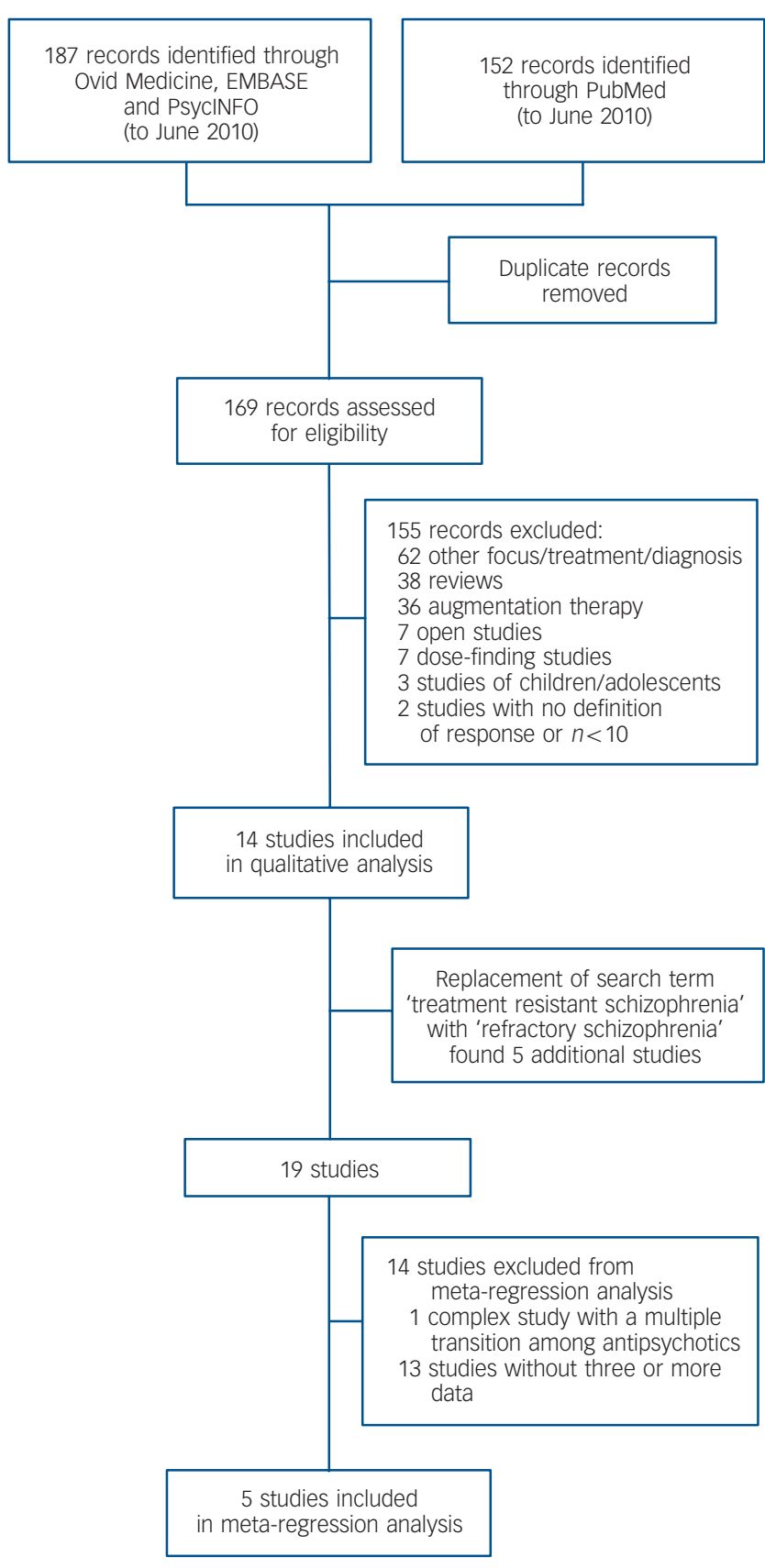

Fig. 1 Literature search.

Thirteen additional studies were excluded on the basis of having two or fewer data reporting points within 6 weeks (Table DS1). A total of five studies that reported on the rating scale scores through week 6 were included for meta-regression (Table DS2). ${ }^{5,13,14-16}$ Each study had two treatment arms and the antipsychotics studied were clozapine (four studies), olanzapine (two), aripiprazole, chlorpromazine, perphenazine and ziprasidone (one study each). Clozapine treatment was gradually increased over several weeks to the target doses, ranging from $100 \mathrm{mg}$ to $900 \mathrm{mg}$. The study samples were predominantly male $(60-80 \%$, where indicated) with an average age of 36-42 years. As expected, the average score on the Clinical Global Impression - Severity (CGI-S) scale was relatively high at 5.2, corresponding to marked illness. Except in one study, ${ }^{5}$ patients with drug resistance and those with intolerance were both recruited; however, the results of these studies were not stratified by resistance or intolerance. With the exception of one study that described continuous data on BPRS four key items and an anergia item, ${ }^{5}$ positive $v$. negative symptoms data were either presented as pre- and post-treatment data, ${ }^{13,14,16}$ or not presented separately. ${ }^{15}$ Affective or cognitive symptoms were not addressed in these studies. All studies adopted a flexible dosing design, and the PANSS was used by all studies except for one that used the BPRS. ${ }^{5}$

\section{Analysis}

Considering all antipsychotics, a first-order autoregressive covariance structure was found to yield the best fit among the various structures tested (five studies, $n=1019$ ). Standardised mean item scores were found to change significantly over the course of the 6 -week period $(F=34.68$, d.f. $=6,22, P<0.0001)$. Bonferroni-adjusted pairwise comparisons found that the baseline scores were significantly higher than the scores observed at all other time points (adjusted $P<0.0001$ ). Week 1 standardised mean item scores were significantly higher than those observed at all subsequent time points $(P<0.0001$ in each case). Week 2 scores were also significantly higher than those observed at weeks 3-6 $(P<0.0001)$. Week 3 scores were significantly higher than scores at weeks $4-6(P=0.00268,0.0086$ and 0.0004 respectively). Week 4 scores were significantly higher than scores at week 6 $(P=0.0172)$ but did not differ from week 5 scores $(P=0.6909)$. Scores at weeks 5 and 6 were not significantly different from each other $(P=0.7679)$. The mean standardised scores at each time point are summarised in Fig. 2.

For clozapine, a heterogeneous first-order autoregressive covariance structure was found to yield the best fit among the various structures tested (four studies, $n=356$ ). Standardised mean item scores were found to change significantly over the course of the study $(F=90.91$, d.f. $=6,18, \quad P<0.0001)$. Bonferroni-adjusted pairwise comparisons found that the baseline scores were significantly higher than the scores observed at all other time points (baseline $v$. week $1 P=0.0177$; baseline $v$. weeks 2-6 $P<0.0001)$. Week 1 scores were significantly higher than those observed at all subsequent time points $(P<0.0001$ in each case). Week 2 scores were also significantly higher than those observed at weeks 3-6 $(P<0.0001)$. Week 3 scores were significantly higher than scores at weeks 4-6 $(P=0.0007$, $<0.0001$ and $<0.0001$ respectively). Week 4 scores were significantly higher than scores at weeks 5 and $6(P=0.0002$ and $<0.0001$ respectively). Week 5 scores were also significantly higher than week 6 scores $(P=0.0003)$. The mean standardised scores at each time point are summarised in Fig. 3.

The mean standardised item scores were 3.4 for all antipsychotics and 3.5 for clozapine, corresponding to a PANSS score of 103. This baseline score was higher than that previously reported for patients with non-resistant schizophrenia by about 10 points. $^{2}$ Overall, a $20 \%$ decrease was noted at week 4 , and a $30 \%$ decrease was not achieved by the end of week 6 for all antipsychotics pooled. In contrast, for clozapine alone a $20 \%$ decrease was noted at week 3 and this increased further to $30 \%$ by week 6 . An improvement of 0.17 for all antipsychotics (29\% of the improvement from baseline through week 6) and 0.27 for clozapine (33\%) was noted in weeks 4-6. Thus, approximately two-thirds of the improvement observed in 6 weeks occurred within the first 3 weeks. Nevertheless, a smaller improvement over the following 3 weeks corresponded to a further 5.1 point decrease in PANSS scores for all antipsychotics and 8.1 points for clozapine.

The results for second-generation antipsychotics other than clozapine (four studies, $n=380$ ) and for completer-only samples (two studies, $n=233)^{13,27}$ are shown in online Figs DS1 and 


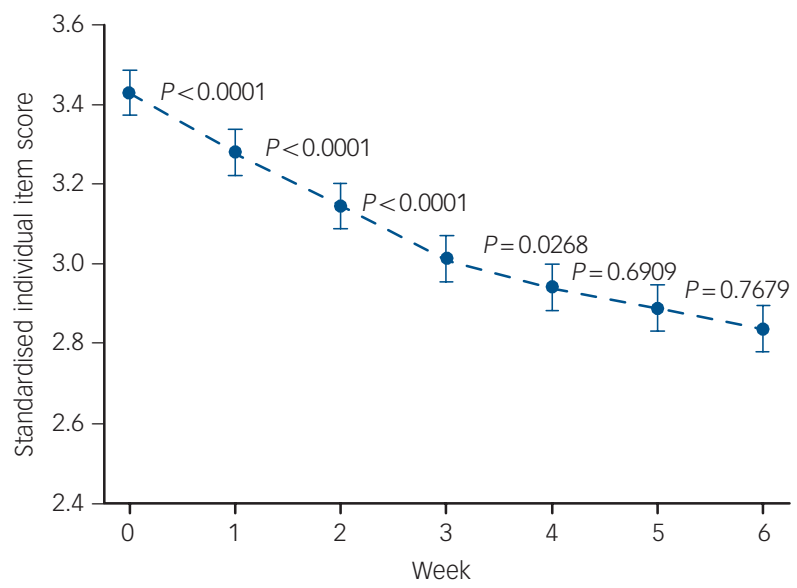

Fig. 2 Standardised individual item mean scores $v$. week for all antipsychotics (five studies, $n=1019$ ). Bars indicate standard error.

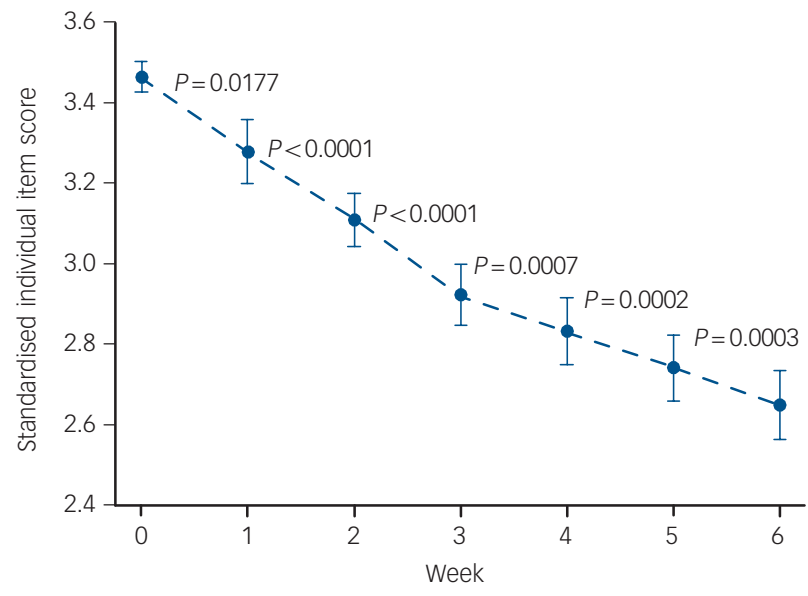

Fig. 3 Standardised individual item mean scores $v$. week for clozapine (four studies, $n=356$ ). Bars indicate standard error
DS2. These data are only preliminary in nature, given the small number of studies and participants.

\section{Discussion}

We were interested in whether improvement with antipsychotic medication might be seen relatively early in treatment-resistant schizophrenia, similar to the early improvement found in patients with non-refractory disease. ${ }^{1,2}$ It would have been desirable if published antipsychotic trials in this population had adopted a uniform definition of treatment resistance and treatment response; however, study definitions as well as trial durations varied considerably, resulting in limited comparability across studies. Adopting the threshold recently proposed by Leucht et al (a $25 \%$ or more decrease in the BPRS or PANSS) ${ }^{17}$ would be expected to result in lower response rates compared with the more typical 20\% cut-off, provided that other absolute criteria were not used (e.g. a post-treatment CGI-S score of 3 or less, or a BPRS score of 35 or less). ${ }^{5}$ This highlights the importance of researchers reaching a consensus on what constitutes 'treatment-resistant schizophrenia' and 'treatment response' thereafter. Moreover, although an evaluation of a third antipsychotic trial for those who have already failed to respond to two prospective antipsychotic trials appears to be ideal, such studies are few, ${ }^{32-34}$ and none has specifically reported on the timing issue. This noted, we evaluated five studies providing at least three LOCF data points within a 6-week treatment period. Preliminary results indicated that over this interval the greatest treatment effect (two-thirds of the improvement) may occur in the first 3 weeks, with more gradual and attenuated improvement in the following weeks. Therefore, prolonging an antipsychotic trial dosed appropriately in a patient with 'treatment resistance' in the absence of response beyond this period might not yield robust benefit, although even attenuated later improvements can still be of clinical significance. Determining the time at which the first trial should be abandoned and a subsequent trial initiated constitutes critical data to guide therapeutics, a question that only recently has begun to be addressed. ${ }^{35}$ This is especially pertinent to patients with treatment-resistant or refractory disorder who are often exposed to high doses and extended antipsychotic trials in an attempt to elicit a clinical response.
The results of our study may argue for caution on a 'wait and watch' approach. However, until we know more about possible differences in trajectory of response in this particular patient subgroup, it would be premature to argue for discontinuation in the face of poor response after the first 3-6 weeks, particularly for clozapine. Moreover, 'treatment-resistant' patients clearly constitute a heterogeneous group, including both patients truly 'resistant' to high doses of antipsychotics (e.g. CPZeq $1000 \mathrm{mg}$ ) and patients with 'refractory' disorder who may be less severely ill but are unable to tolerate such high doses. In fact, it is possible that the initial steeper decline in clinical scores is primarily driven by the latter group. ${ }^{36}$ In this respect, resistant and intolerant cases may need to be described separately, although most of the past studies have failed to do so.

\section{Limitations of the study}

Limitations of this investigation include the small number of studies available for analysis as well as the heterogeneous and non-standardised nature of the sample, although all patients were classified as having treatment-resistant/refractory schizophrenia according to each study. Specifically, a paucity of available data points precluded quantitative analysis and within-patient trajectory analysis of response, as well as examination of dose response for individual antipsychotics. The latter is particularly pertinent to clozapine with regard to initial titration, final dose and drug level at steady state. ${ }^{37}$ More consistent improvement with clozapine over 6 weeks may be a product of more gradual titration with this medication at an earlier treatment stage. Further, some data required extrapolation from figures accompanying the published reports, although a past work was obliged to use this strategy as well. ${ }^{1}$ We restricted the search to double-blind studies, which is consistent with previous work involving samples with non-refractory disorder, ${ }^{1,2}$ but limited the number of studies analysed. A distinction between completer and ITT results is important: LOCF data were available for five studies, whereas only two provided data for completers. The use of LOCF, a standard method in clinical trials to deal with participants leaving the study at various time points in the follow-up period of an intervention study, represents a compromise by including data from both completer and 
premature attrition groups. Results should be interpreted with caution in this regard; although analysing the data for the completers-only group has the potential of overestimating early antipsychotic effects, LOCF data may have the opposite effect. The results described herein should therefore be regarded as preliminary. Finally, it is important to acknowledge that relevant outcomes in the longer term are no longer confined to improvements in symptomatic scores and should be extended to other relevant areas. ${ }^{38-40}$ Furthermore, a possibility remains largely unaddressed that the speed of improvement differs according to symptoms or illness domains, although data presentation has not usually been stratified in this respect. Also pertinent to note is a recent indication that functional outcome may not be so delayed. ${ }^{41}$

\section{Future research}

To better address the time-line issue it is desirable that future studies include longitudinal data on response rates as established by a priori criteria, e.g. $20 \%, 30 \%, 50 \%$ or $25 \%$ (in accordance with Leucht et al), ${ }^{17}$ at each available assessment point, the average score of the primary outcome across time, median/mean time to response among responders, and time-line by which a certain percentage of patients are classified as responders, to allow for a better understanding of a reasonable antipsychotic trial for challenging patients in clinical practice. The data are better presented with ITT populations and observed cases at each assessment. Finally, such data can be interpreted in the context of the value of early improvement in predicting later response and the trajectory in response $e^{42,43}$ as well as identifying persistence on response status on the individual level, ${ }^{44}$ two important issues in the management of patients who are considered to have treatment-resistant disorder.

To conclude, definition of treatment-resistant schizophrenia and treatment response needs to be carefully delineated, in combination with more detailed data presentation throughout the treatment period. Limited evidence from studies longer than 6 weeks suggests that the greatest treatment effect is within 3-4 weeks. This is in line with what has been reported for non-refractory patients, but does not necessarily rule out a delayed onset of antipsychotic action specifically for clozapine. Future studies need to monitor response earlier in the course of treatment, whereas longer-term investigations can evaluate the value of early response in predicting later outcome. It may well be that there are different trajectories of response, and distinguishing these will take us a step forward in defining subgroups, information that would have important clinical implications.

\footnotetext{
Takefumi Suzuki, MD, Centre for Addiction and Mental Health, Geriatric Mental Health Program and Multimodal Imaging Group, and Department of Psychiatry, University of Toronto, Ontario, Canada, and Keio University School of Medicine, Department of Neuropsychiatry, Tokyo, Japan; Gary Remington, FRCPC, Department of Psychiatry, University of Toronto, and Centre for Addiction and Mental Health Schizophrenia Program, Toronto, Ontario, Canada; Tamara Arenovich, MSC, Centre for Addiction and Mental Health, Clinical Research Department, Biostatistical for Addiction and Mental Health, Clinical Research Department, Biostatistical
consulting Service, Toronto, Ontario, Canada; Hiroyuki Uchida, MD, Centre for Addiction and Mental Health, Geriatric Mental Health Program, Toronto, Ontario, Canada, and Keio University School of Medicine, Department of Neuropsychiatry, Tokyo, Japan; Ofer Agid, MD, Department of Psychiatry, University of Toronto, and Centre for Addiction and Mental Health, Schizophrenia Program, Toronto, Ontario; Ariel Graff-Guerrero, MD, Department of Psychiatry, University of Toronto, and Centre for Addiction and Mental Health, Multimodal Imaging Group, Toronto, Ontario; David C. Mamo, FRCPC, Centre for Addiction and Mental Health, Geriatric Mental David C. Mamo, FRCPC, Centre for Addiction and Mental Health, Geriatric Mental
Health Program, Toronto, Department of Psychiatry, University of Toronto, and Centre for Addiction and Mental Health, Multimodal Imaging Group, PET Centre, Toronto, Ontario, Canada
}

Correspondence: David C. Mamo, Geriatric Mental Health Program, Centre for Addiction and Mental Health, 1001 Queen Street West, Toronto, Ontario M6J 1H4, Canada. Email: david_mamo@camh.net

First received 18 Jun 2010, final revision 13 Jan 2011, accepted 21 Mar 2011

\section{Acknowledgements}

The authors thank Dr Istvan Bitter and Eli Lilly for generously sharing the data, and Dr John Kane for his thoughtful input.

\section{References}

1 Agid O, Kapur S, Arenovich T, Zipursky RB. Delayed-onset hypothesis of antipsychotic action: a hypothesis tested and rejected. Arch Gen Psychiatry 2003; 60: 1228-35.

2 Sherwood M, Thornton AE, Honer WG. A meta-analysis of profile and timecourse of symptom change in acute schizophrenia treated with atypical antipsychotics. Int J Neuropsychopharmacol 2006; 9: 357-66.

3 Meltzer HY. Treatment-resistant schizophrenia - the role of clozapine. Curr Med Res Opin 1997; 14: 1-20.

4 Leucht S, Corves C, Arbter D, Engel RR, Li C, Davis JM. Second-generation versus first-generation antipsychotic drugs for schizophrenia: a metaanalysis. Lancet 2009; 373: 31-41.

5 Kane J, Honigfeld G, Singer J, Meltzer H. Clozapine for the treatment-resistant schizophrenic. A double-blind comparison with chlorpromazine. Arch Gen Psychiatry 1988; 45: 789-96.

6 Stern RG, Kahn RS, Davidson M, Nora RM, Davis KL. Early response to clozapine in schizophrenia. Am J Psychiatry 1994; 151: 1817-8.

7 Rosenheck R, Evans D, Herz L, Cramer J, Xu W, Thomas J, et al. How long to wait for a response to clozapine: a comparison of time course of response to clozapine and conventional antipsychotic medication in refractory schizophrenia. Schizophr Bull 1999; 25: 709-19.

8 Conley RR, Carpenter WT, Tamminga CA. Time to clozapine response in a standardized trial. Am J Psychiatry 1997; 154: 1243-7.

9 Meltzer HY, Bastani B, Kwon KY, Ramirez LF, Burnett S, Sharpe J. A prospective study of clozapine in treatment-resistant schizophrenic patients. I. Preliminary report. Psychopharmacology (Berl) 1989; 99 (suppl): S68-72.

10 Meltzer HY. Duration of a clozapine trial in neuroleptic-resistant schizophrenia. Arch Gen Psychiatry 1989; 46: 672.

11 Zito JM, Volavka J, Craig TJ, Czobor P, Banks S, Vitrai J. Pharmacoepidemiology of clozapine in 202 inpatients with schizophrenia. Ann Pharmacother 1993; 27: 1262-9.

12 Buchanan RW, Kreyenbuhl J, Kelly DL, Noel JM, Boggs DL, Fischer BA, et al. The 2009 schizophrenia PORT psychopharmacological treatment recommendations and summary statements. Schizophr Bull 2010; 36: 71-93.

13 Tollefson GD, Birkett MA, Kiesler GM, Wood AJ. Double-blind comparison of olanzapine versus clozapine in schizophrenic patients clinically eligible for treatment with clozapine. Biol Psychiatry 2001; 49: 52-63.

14 Bitter I, Dossenbach MR, Brook S, Feldman PD, Metcalfe S, Gagiano CA, et al. Olanzapine versus clozapine in treatment-resistant or treatment-intolerant schizophrenia. Prog Neuropsychopharmacol Biol Psychiatry 2004; 28 : $173-80$.

15 Kane JM, Meltzer HY, Carson WH, McQuade RD, Marcus RN, Sanchez R. Aripiprazole for treatment-resistant schizophrenia: results of a multicenter randomized, double-blind, comparison study versus perphenazine. J Clin Psychiatry 2007; 68: 213-23.

16 Sacchetti E, Galluzzo A, Valsecchi P, Romeo F, Gorini B, Warrington L. Ziprasidone vs clozapine in schizophrenia patients refractory to multiple antipsychotic treatments: the MOZART study. Schizophr Res 2009; 113: 112-21.

17 Leucht S, Davis JM, Engel RR, Kissling W, Kane JM. Definitions of response and remission in schizophrenia: recommendations for their use and their presentation. Acta Psychiatr Scand Suppl 2009; 438: 7-14.

18 Meltzer HY, Bobo WV, Roy A, Jayathilake K, Chen Y, Ertugrul A, et al. A randomized, double-blind comparison of clozapine and high-dose olanzapine in treatment-resistant patients with schizophrenia. J Clin Psychiatry 2008; 69: $274-85$

19 Kane JM, Khanna S, Rajadhyaksha S, Giller E. Efficacy and tolerability of ziprasidone in patients with treatment-resistant schizophrenia. Int Clin Psychopharmacol 2006; 21: 21-8.

20 Lal S, Thavundayil JX, Nair NP, Annable L, Ng Ying Kin NM, Gabriel A, et al. Levomepromazine versus chlorpromazine in treatment-resistant schizophrenia: a double-blind randomized trial. J Psychiatry Neurosci 2006; 31: 271-9.

21 Conley RR, Kelly DL, Nelson MW, Richardson CM, Feldman S, Benham R, et al. Risperidone, quetiapine, and fluphenazine in the treatment of patients with therapy-refractory schizophrenia. Clin Neuropharmacol 2006; 28: 163-8.

22 Kane JM, Marder SR, Schooler NR, Wirshing WC, Umbricht D, Baker RW, et al. Clozapine and haloperidol in moderately refractory schizophrenia: a 6-month 
randomized and double-blind comparison. Arch Gen Psychiatry 2001; 58 965-72.

23 Zhang XY, Zhou DF, Cao LY, Zhang PY, Wu GY, Shen YC. Risperidone versus haloperidol in the treatment of acute exacerbations of chronic inpatients with schizophrenia: a randomized double-blind study. Int Clin Psychopharmacol 2001; 16: 325-30

24 Breier A, Hamilton SH. Comparative efficacy of olanzapine and haloperidol for patients with treatment-resistant schizophrenia. Biol Psychiatry 1999; 45: 403-11.

25 Wirshing DA, Marshall BD, Green MF, Mintz J, Marder SR, Wirshing WC Risperidone in treatment-refractory schizophrenia. Am J Psychiatry 1999; 156: 1374-9.

26 Bondolfi G, Dufour H, Patris M, May JP, Billeter U, Eap CB, et al. Risperidone versus clozapine in treatment-resistant chronic schizophrenia: a randomized double-blind study. The Risperidone Study Group. Am J Psychiatry 1998; 155: 499-504.

27 Conley RR, Tamminga CA, Bartko JJ, Richardson C, Peszke M, Lingle J, et al. Olanzapine compared with chlorpromazine in treatment-resistant schizophrenia. Am J Psychiatry 1998; 155: 914-20.

28 Hong CJ, Chen JY, Chiu HJ, Sim CB. A double-blind comparative study of clozapine versus chlorpromazine on Chinese patients with treatmentrefractory schizophrenia. Int Clin Psychopharmacol 1997; 12: 123-30.

29 Rosenheck R, Cramer J, Xu W, Thomas J, Henderson W, Frisman L, et al. A comparison of clozapine and haloperidol in hospitalized patients with refractory schizophrenia. Department of Veterans Affairs Cooperative Study Group on Clozapine in Refractory Schizophrenia. N Engl J Med 1997; 337: 809-15.

30 Breier A, Buchanan RW, Kirkpatrick B, Davis OR, Irish D, Summerfelt A, et al. Effects of clozapine on positive and negative symptoms in outpatients with schizophrenia. Am J Psychiatry 1994; 151: 20-6.

31 Kinon BJ, Kane JM, Johns C, Perovich R, Ismi M, Koreen A, et al. Treatment of neuroleptic-resistant schizophrenic relapse. Psychopharmacol Bull 1993; 29 309-14.

32 Agid O, Remington G, Kapur S, Arenovich T, Zipursky RB. Early use of clozapine for poorly responding first-episode psychosis. J Clin Psychopharmacol 2007; 27: 369-73.

33 Suzuki T, Uchida $\mathrm{H}$, Watanabe $\mathrm{K}$, Nomura K, Takeuchi $\mathrm{H}$, Tomita M, et al. How effective is it to sequentially switch among olanzapine, quetiapine and risperidone? A randomized, open-label study of algorithm-based antipsychotic treatment to patients with symptomatic schizophrenia in the real-world clinical setting. Psychopharmacology (Berl) 2007; 195: 285-95.

34 Stroup TS, Lieberman JA, McEvoy JP, Davis SM, Swartz MS, Keefe RS, et al. Results of phase 3 of the CATIE schizophrenia trial. Schizophr Res 2009; 107 1-12.

35 Kinon BJ, Chen L, Ascher-Svanum H, Stauffer VL, Kollack-Walker S, Zhou W, et al. Early response to antipsychotic drug therapy as a clinical marker of subsequent response in the treatment of schizophrenia. Neuropsychopharmacology 2010; 35: 581-90.

36 Lieberman JA, Safferman AZ, Pollack S, Szymanski S, Johns C, Howard A, et al. Clinical effects of clozapine in chronic schizophrenia: response to treatment and predictors of outcome. Am J Psychiatry 1994; 151: 1744-52.

37 Schulte $P$. What is an adequate trial with clozapine? Therapeutic drug monitoring and time to response in treatment-refractory schizophrenia. Clin Pharmacokinet 2003; 42: 607-18.

38 Suzuki T, Uchida $\mathrm{H}$, Watanabe $\mathrm{K}$, Kashima $\mathrm{H}$. Treatment target in schizophrenia: a critical review and a clinical suggestion. Psychopharmacol Bull 2008; 41: 80-102.

39 Wheeler A, Humberstone V, Robinson G. Outcomes for schizophrenia patients with clozapine treatment: how good does it get? J Psychopharmacol 2009; 23: 957-65.

40 Remington G, Foussias G, Agid O. Progress in defining optimal treatment outcome in schizophrenia. CNS Drugs 2010; 24: 9-20.

41 Kinon BJ, Chen L, Ascher-Svanum H, Stauffer VL, Kollack-Walker S, Zhou W, et al. Challenging the assumption that improvement in functional outcomes is delayed relative to improvement in symptoms in the treatment of schizophrenia. Schizophr Res 2010; 118: 176-82.

42 Leucht S, Shamsi SA, Busch R, Kissling W, Kane JM. Predicting antipsychotic drug response - replication and extension to six weeks in an international olanzapine study. Schizophr Res 2008; 101: 312-9.

43 Levine SZ, Leucht S. Elaboration on the early-onset hypothesis of antipsychotic drug action: treatment response trajectories. Biol Psychiatry 2010; 68: 86-92.

44 Glick ID, Bossie CA, Alphs L, Canuso CM. Onset and persistence of antipsychotic response in patients with schizophrenia. J Clin Psychopharmacol 2009; 29: 542-7. 